\title{
Transnationale Infrastrukturen des Humanen
}

Technologien als Mittel gesellschaftlicher Autopoiesis

STEFAN BECK

In dem folgenden Kapitel wird gefragt, wie sich aus der Perspektive der Science and Technology Studies die Nutzung der Biomedizin untersuchen lässt. Hierfür werden drei Konzepte vorgestellt: der Begriff des Dispositivs von Michel Foucault, der Begriff der Plattform, wie er von Peter Keating und Alberto Cambrosio eingeführt wurde, und schließlich das Konzept der Infrastruktur, das von Susan Leigh Star und Karen Ruhleder vorgeschlagen wurde. Alle drei Konzepte machen deutlich, dass Technik und Technologie stets als eingebettet in einem Netzwerk zu untersuchen ist, das unterschiedliche Wirklichkeitsbereiche miteinander verbindet: etwa ökonomische Kalküle, moralische Motive, wissenschaftliche Überzeugungen und Machtbeziehungen. Die drei Begriffe verdeutlichen zudem, dass für die Nutzer die Biomedizin und die komplexen Netzwerke aus Wissen, Dingen, Institutionen und Akteuren zum Zeitpunkt ihrer Nutzung nur teilweise sichtbar sind. Hierfür wird der Begriff der »Transparenz« eingeführt: er verweist auf die unproblematische "Gegebenheit", das Nicht-Problematisch-Werden von Technologien im Rahmen normaler Nutzung. Der Begriff der Transparenz ermöglicht aber auch, besser zu verstehen, wann Technik und Technologie problematisch werden. Dieser Zusammenhang wird am Beispiel einer empirischen Untersuchung eines zypriotischen Programms zur Behandlung von Leukämie-Patienten erläutert. 


\section{Weiterführende Literatur}

Hayden, Cori (2003): When Nature Goes Public. The Making and Unmaking of Bioprospecting in Mexico. Princeton: Princeton University Press. Auf Grundlage einer umfangreichen Ethnographie in Mexiko und den USA untersucht Hayden "Bioprospecting" - die Suche von Wissenschaftlern der „Ersten Welt« nach neuen pharmakologisch wirksamen Substanzen in der "Dritten Welt" - als eine komplexe, fragile und moralisch hoch umstrittene Praxis, die wissenschaftliches Wissen und »indigenes Wissen «, unterschiedliche Interessen, moralische Ökonomien und Politiken (mit großen wie kleinem P) in einer neuen globalen Form verbindet. Die Studie greift verschiedene Ansätze der STS auf und entwickelt sie für eine Analyse globaler Machtbeziehungen und »lokaler« Effekte weiter.

Sanal, Aslihan (2011): New Organs Within Us. Transplants and the Moral Economy. Durham, London: Duke University Press.

Die Transplantationsmedizin wird von Sanal auf der Grundlage intensiver ethnographischer Forschung vor allem in der Türkei als global verfügbare, soziale Form interpretiert, die neue Relationen stiftet - zwischen Wissenschaftlern, Medizinern und Patienten, zwischen Wissenschaft und Ökonomie, zwischen Patienten und Organspendern, zwischen (trans-)nationalen Regulationen und »eingeborenen" Moralitäten. Die Studie greift einige STS-Ansätze auf, gewinnt ihre Stärke aber vor allem durch die Perspektive der kritischen Medizinanthropologie - sie kann als Beispiel für das neue Schnittfeld zwischen STS und Medical Anthropology herangezogen werden.

Fullwiley, Duana (2011): The Enculturated Gene. Sickle Cell Politics and Biological Difference in West Africa. Princeton University Press.

Die Studie analysiert auf Basis einer Ethnographie in Westafrika, Frankreich und den USA wie medizinische Konzepte und Praktiken auf der einen und "volkstümliche", »laienhafte" Konzepte der Sichelzell-Anämie auf der anderen Seite neue Assoziationen zwischen Patienten, zwischen Wissenschaft und Alltag sowie zwischen Gesundheitspolitik und Ökonomie herausbilden. Im Zentrum stehen Fragen, wie Wissen verkörpert wird, welche Subjektivitäten sich durch die Annahme verschiedenen Wissens ergeben und wie diese neuen sozialen Formen in Globalisierungsprozesse eingebettet sind. 


\section{Einleitung}

Was ist die Aufgabe der Europäischen Ethnologie und der Sozial- und Kulturanthropologie im Feld der Wissenschafts- und Technikforschung? Zieht man die umfassende Fach-Definition der »American Anthropological Association« heran, dann ergibt sich nicht gerade ein bescheidenes Frageprogramm: »Anthropology is the study of humans, past and present. To understand the full sweep and complexity of cultures across all of human history, anthropology draws and builds upon knowledge from the social and biological sciences as well as the humanities and physical sciences.« Obwohl nicht ausdrücklich erwähnt, ist offensichtlich, dass die Menschheit in all ihrer Diversität und historischen Entwicklung ohne Wissen darum, wie sie zu unterschiedlichen Zeiten und an unterschiedlichen Orten Wissenschaft und Technik nutzt(e), nicht recht verstanden werden kann: Denn indem Menschen Wissen und Technik nicht nur produzieren, sondern in Gesellschaften auch systematisch auf sich selbst anwenden, entsteht erst das Erkenntnisobjekt der Anthropologie. Damit sind zwei Zusammenhänge impliziert: Erstens darf Technik nicht auf sichtbare und vergegenständlichte »Dinge« eingegrenzt werden, mit denen sich ein überschaubarer, transparenter und in seinen Folgen überschaubarer Umgang pflegen ließe. Eine $\mathrm{zu}$ einfache, überwiegend materialistisch ausgerichtete Epistemologie gegen-über komplexen technologischen Artefakten verfehlt ihre analytischen Aufgaben. Zweitens ist es offensichtlich, dass Technik keine »sozialen Folgen« in einem einfachen Sinne hat: Wissenschaft und Technik müssen als Teil gesellschaftlicher Selbsteinwirkung und als Motor sozialer wie kultureller Selbstreflektion interpretiert werden. Als ein konstitutiver Teil von sozialen Praktiken formieren sie Kultur ebenso wie die sie äußere und innere menschliche »Natur« und Selbstverständnisse transformieren helfen.

Anhand von zwei Beispielen aus dem medizinischen Bereich erläutert das Kapitel den prinzipiell für unterschiedliche Zwecksetzungen offenen, nicht deterministischen Charakter wissenschaftlich-technologischer Innovationen: 1. am Beispiel der Möglichkeit, durch genetische Tests Vorhersagen über Krankheitsdispositionen zu machen und dadurch Gruppen von Menschen zu klassifizieren; und 2. an der Arbeit einer transnational tätigen Biobank zur Behandlung von Leukämie-Kranken. In beiden Fällen werden die Optionen der Technologie vor dem Hintergrund kultureller Relevanzset- 
zungen und sozialer wie politischer Konstellationen eingesetzt - um diesen Zusammenhang analysieren zu können, werden drei theoretisch ähnlich gelagerte Begriffe vorgestellt - Dispositiv, Plattform und Infrastruktur -, die im Feld der STS mit einigem Erfolg eingesetzt werden.

\section{Genetische Transparenz und die Herstellung VON POPULATIONEN 2.0}

Am zehnten November 2005 veröffentlichte ein Autorenteam aus isländischen und US-amerikanischen Humangenetikern in der Fachzeitschrift »Nature Genetics« (Helgadottir et al. 2005) Ergebnisse eines mehrjährigen Forschungsprojektes, die aus gleich mehreren Gründen für einigen Wirbel in der internationalen Tagespresse sorgten. Das Aufsehen erregendste Ergebnis der Studie war dabei nicht, dass bei isländischen Patienten ein Gen entdeckt worden war, das bei den jeweiligen TrägerInnen mit einem leicht erhöhten Risiko für die Entstehung von Herzinfarkten korreliert ist. ${ }^{1}$ Sensationell erschien vielmehr in der nicht-wissenschaftlichen Rezeption der Befund, dass dieses in der isländischen Bevölkerung ebenso wie in anderen europäischen Bevölkerungen recht verbreitete Gen bei Menschen mit gemischter afrikanischer und europäischer Herkunft - wie dies vor allem bei vielen Afro-Amerikanern in den USA der Fall ist - zu einem mehr als dreifach erhöhten Risiko führt, einen Herzinfarkt zu erleiden.

Diese Studie geht wesentlich auf Befunde einer Arbeitsgruppe der isländischen Firma deCode Genetics zurück, einem privatwirtschaftlich finanzierten Forschungsinstitut, das auf der Grundlage einer Kooperation mit dem isländischen Staat nach Genen sucht, von denen angenommen wird, dass sie zur Entwicklung von weit verbreiteten »Volks«-Krankheiten, wie etwa Asthma, Herzinfarkt oder Krebs beitragen. (Pálsson/Rabinow 1999) Für dieses sehr ambitioniert angelegte Forschungsprogramm kombiniert die

1 Das Gen, das den Namen HapK erhielt, codiert ein Eiweiß, dass an einem biochemischen Stoffwechselpfad beteiligt ist, der Entzündungsprozesse im Körper verstärkt, die wiederum zu einem Aufbrechen von Fettablagerungen in den Arterien führen können, die - in einem weiteren Schritt - Herzkranzgefäße verstopfen und zu Infarkten führen können. 
Firma drei unabhängige Datenquellen, die in dieser Kombination nur in Island verfügbar sind: Erstens individuelle Krankheitsgeschichten, die durch die öffentliche Gesundheitsverwaltung Islands in den vergangenen Jahrzehnten in Form von Patienten-Akten gespeichert wurden, zweitens Informationen über die Familien- und Abstammungsverhältnisse der ausgewählten Kranken, wobei auf genealogische Datenbanken zurückgegriffen wird, die in jahrzehntelanger Kleinarbeit von isländischen Hobby-Genealogen zusammengestellt wurden, und drittens hochmoderne genetische Diagnoseverfahren, mit denen gezielt genetische Mutationen und Variabilitäten bei Kranken gesucht werden. Ziel dieser Integration heterogener Datenquellen ist es, einen statistischen Zusammenhang zwischen genetischen, in Familien gehäuft auftretenden Varianzen und gesundheitlichen Risiken zu demonstrieren. (Pálsson/Rabinow 2001) Die Verlässlichkeit der Analysen erhöht sich dabei durch die historische Tiefe des verfügbaren Datenmaterials aus Patientengeschichten, Familienstammbäumen und weiteren populationsgenetischen Analysen bedeutend. (Helgason et al. 2000; Pálsson/Harđardóttir 2002)

Das weiter gesteckte Ziel der Firma deCode Genetics besteht dabei darin, auf der Basis dieses Wissens neuartige Diagnoseverfahren, vor allem aber neue Medikamente zu entwickeln, die die molekularbiologischen $U r$ sachen und nicht mehr nur - wie in der klassischen, medizinischen Praxis die organischen Symptome von Volkskrankheiten wie Diabetes, Asthma, Bluthochdruck oder Herzinfarkte bekämpfen. Dieses auch international viel diskutierte und vor allem wegen der kommerziellen Nutzung von höchst intimen Gesundheitsdaten schon seit längerem viel kritisierte Programm (Rose 2001; Sigurdsson 2001) sieht vor, dass die isländische Bevölkerung für die Überlassung ihrer Gesundheitsdaten an die Privatfirma deCode Genetics einen privilegierten und vor allem verbilligten Zugang zu denjenigen Medikamenten erhält, die auf der Grundlage dieser Studien möglicherweise entwickelt werden.

Die überraschende Ironie dieser unter der schreierischen Überschrift »heart risk gene hits African Americans hardest« (Vince 2005) popularisierten Forschungsergebnisse ist dabei - für humangenetische Studien allerdings nicht unüblich - ihre überwiegende Folgenlosigkeit: Weder ist ein Medikament in Sicht, mit dem ein erhöhtes Herzinfarktrisiko reduziert werden könnte, noch hat die Studie wirklich Relevanz für die Isländer, die mit ihren Daten diese Forschungen erst ermöglichten. Der so verlockend 
erscheinende deal, im Gegenzug zur Bereitschaft, dem Unternehmen die Gesundheitsdaten zur Verfügung zu stellen, eine Art ethnisches Vorkaufsrecht für die neuesten Medikamente zu erhalten, geht zumindest in diesem Fall nicht auf: Das menschliche Genom ist zu universell, als dass »ethnische Demarkationsversuche « erfolgreich sein könnten. ${ }^{2}$ Stattdessen belegen die Ergebnisse, dass die Isländer - wenn auch möglicherweise unfreiwillig oder unwissend - durch die Firma deCode Genetics zu einem Element eines globalen Netzwerkes biomedizinischer Invention und Intervention gemacht wurden: Ein Sachverhalt, der sich mit Gísli Pálsson und Paul Rabinow (2005) auch positiv interpretieren lässt als die Partizipation an emergenten »transnationalen Märkten ziviler Tugend«, die sich als die >andere Seite< ökonomischer Globalisierung entwickeln.

Doch zurück zu den schwer interpretierbaren Forschungsergebnissen der deCode-Studie: Es ist offensichtlich, dass es nicht das "Gen« ist, dass die Herzinfarkte auslöst - wie in den Überschriften der internationalen Presse ebenso prägnant wie falsch dargestellt -, sondern es ist das entsprechende Genprodukt, das offenbar in den Stoffwechselpfaden unterschiedlicher Populationen auf unterschiedliche Weise wirkt. Es ist mithin das komplexe Zusammenspiel des unterschiedlichen genetischen Hintergrundes des individuellen Stoffwechsels und einer sehr unterschiedlichen Umwelt, das bestimmt, ob das Risiko für einen Herzinfarkt erhöht ist oder nicht. ${ }^{3}$ Es

2 Selbst im Fall der vermeintlich so homogenen isländischen Bevölkerung gilt, dass eine hohe Heterogenität der Abstammungslinien festgestellt werden muss, die auf eine große Zahl von verschiedenen Verbindungen zum Europäischen Festland hindeuten, die in Zuge der Besiedlungsgeschichte Islands in einer spezifischen Form aggregiert wurden. So stellten etwa Helgason et al. auf der Grundlage umfangreicher Analysen isländischer mitochondrialer DNA fest: »The last 1,100 years of mtDNA evolution in Europe have principally been a history of lineage redistribution, within populations because of drift and between populations because of migration. The settlement of Iceland was equivalent to a migrational sampling event of existing genetic diversity at one or more locations in Europe. The subsequent genetic history of the Icelanders has primarily involved stochastic sampling of these founder lineages between generations (interspersed with a small number of mutation events)«. (Helgason et al. 2000: 1012)

$3 \mathrm{Vgl}$. zu analogen Befunden bzgl. der bei unterschiedlichen Menschen sehr verschiedenen Wirkungen des Koffeins auf Herzinfarkt-Risiken die Studie einer Kanadischen Forschergruppe, die schlussfolgert, dass Ernährungsstudien nur bedeutsam sind, wenn sie mögliche genetische Diversität in Rechnung stellen. (Cornelis 
liegt daher nahe, diese Pressemeldung schnell in die lange Kette der populären, einem platten genetischen Determinismus huldigenden Verlautbarungen unter der Rubrik »Gen-für-XY-entdeckt« einzuordnen. Aber es ist eben durchaus auch in der Linie konventioneller, nicht weniger platter Technikkritik, diese Meldung vor allem als weiteres Beispiel für die potentiellen, negativen Effekte der neuen Genetik zu interpretieren und die potentiell ethnisierenden, essentialisierenden oder gar neo-rassistischen Wirkungen solcher biologischer Klassifikationen anzuprangern (KLASSIFIKATIONEN). Es wird befürchtet, dass hier Populationen 2.0 erzeugt werden, Gruppen von Menschen, in deren Leben - verstanden als Biologie plus Lebensstil - eingegriffen werden kann.

Doch auch fern solcher ebenso simplen wie falschen Erfolgsmeldungen oder Skandalisierungen ist es natürlich alles andere als harmlos, wenn mit afro-amerikanischen Herzinfarkt-Patienten ausgerechnet eine sozial und ökonomisch bereits vielfach benachteiligte Gruppe, die aufgrund ihrer schlechten Lebensbedingungen ein erhöhtes Risiko für HerzkreislaufErkrankungen aufweist, nun auch noch als Träger einer riskanten genetischen Mutation ausgemacht wird. Aber selbst wenn sich aus der Feststellung einer solchen Korrelation eine mögliche Stigmatiserung ergeben könnte, ist noch nicht entschieden, welche sozialen Effekte dies zeitigen kann. Denn diese Mechanismen einer negativen sozialen Klassifikation legen es auch nahe, dass sich die Betroffenen selbst politisch zusammen schließen, um sich gegen eine drohende Benachteiligung zur Wehr zu setzen. Für solche Prozesse der Fremd- wie Selbstklassifikation prägte der amerikanische Kulturanthropologe Paul Rabinow bereits 1992 den Begriff der Biosozialität, um auf die »Bildung neuer kollektiver und individueller Identitäten« hinzuweisen, die sich in den USA nach dem Muster der dort schon seit langem bestehenden Patientengruppen zu formieren begannen. Wie Rabinow in ironischer Pointierung formulierte:

et al. 2006) Mit dem gleichen Recht ließe sich deshalb auch argumentieren, dass es der diffuse genetische Hintergrund oder besser: dass bislang unbekannte Faktoren des Stoffwechsel-Interaktoms dafür verantwortlich sind, ein erhöhtes Herzinfarkt-Risiko zu bedingen. Ein solcher Befund wäre jedoch kaum Schlagzeilengängig und würde kaum die Aufnahme von Risiko-Kapital am internationalen Finanzmarkt erleichtern. 
»Man [kann] sich soziale Gruppen vorstellen, die sich um Chromosom 17, Lokus 16.256, Position 654.376 und Allele mit Guanin-Vertauschung bilden. Solche Gruppen werden über medizinische Spezialisten, Labors, Geschichten und Traditionen ebenso verfügen wie über eine ganze Anzahl pastoraler Betreuer, die ihnen behilflich sein werden, ihr Schicksal zu erfassen, zu teilen, zu beeinflussen und zu >verstehen «. (Rabinow 2004: 143)

In diesem Sinne könnte tatsächlich spekuliert werden, ob sich eine Vereinigung von Betroffenen bilden wird, die die Interessen der vor allem von dem »Herzinfarkt-Gen« betroffenen Afro-Amerikaner gegenüber dem Gesundheitssystem vertritt - etwa um eine frühzeitige Diagnose über die Krankenkassen zu finanzieren - oder um bei der Pharmaindustrie LobbyArbeit zu betreiben mit dem Ziel, nicht nur die Entwicklung von Medikamenten zu beschleunigen, die diese Risiken minimieren, sondern vor allem die Lebensbedingungen der Betroffenen nachhaltig $\mathrm{zu}$ verbessern. Denn natürlich besteht durchaus die Gefahr, dass das erhöhte Herzinfarktrisiko von Afro-Amerikanern nun nicht mehr vorwiegend mit schlechten Lebensbedingungen erklärt wird; mit Umweltfaktoren, die folglich als dringend der Veränderung bedürftig erscheinen. Werden dagegen die Krankheitsursachen überwiegend genetisch erklärt, besteht die Möglichkeit, dass die Prävention damit aus dem Bereich des sozialpolitisch Veränderbaren implizit entfernt wird und stattdessen die Behandlung der Symptome in den Kompetenzbereich der Medizin abgeschoben wird. ${ }^{4}$ Im hier diskutierten Zusammenhang ist allerdings entscheidend, dass diese Identitäts- und Gruppenbildungseffekte von Rabinow aus einer Perspektive analysiert werden, die keinen deterministischen Zusammenhang von Tech-

4 Vgl. kritisch zu den Mechanismen einer »Genetisierung« von Herz-KreislaufErkrankungen die Studien des Kulturgeographen Edward Hall (2004, 2005). Hall analysiert die heterogenen Netzwerke der Wissensproduktion und arbeitet heraus, wie unterschiedliche, konkurrierende Erklärungsansätze etwa aus einer sozialmedizinischen, einer klassisch medizinischen oder einer biomedizinischen Perspektive (Herzinfarkt als Ergebnis »riskanten Lebensstils«, als Krankheit sozialer Ungleichheit, als Ergebnis genetischer Disposition) zu einer tentativen, stets bestreitbaren wissenschaftlichen Hypothese: »Herz-Kreislauf-Erkrankungen besitzen eine genetische Komponente« amalgamiert werden. »Genetisierung« wird in dieser wissensanthropologischen Studie als unabgeschlossener Integrationsversuch widerstreitender Epistemologien rekonstruiert. 
nik und ihren »Folgen« annimmt. Entsprechend behauptet er nicht einfach, dass mit der Neuen Genetik eine neue machtvolle Klassifikationsmaschinerie in die Welt komme, die - ähnlich wie die Alte Genetik und die aus ihr entwickelte Eugenik oder ein genetisch argumentierender Rassismus - Gruppen willkürlich konstruiere und Menschen in diese Schubladen stecke. Sondern Rabinow analysiert zutreffend jene sozialen und kulturellen Mechanismen, über die die Neue Genetik daran beteiligt sei, der Menschheit neue Mittel der Selbsteinwirkung und -erschaffung (Autopoiesis) zur Verfügung zu stellen; es entstehe momentan »eine neue Gestalt von [gesellschaftlicher] Autopoiesis, die ich >Biosozialität Handelte es sich bei der [alten] Soziobiologie um eine Form von Kultur, die auf der Grundlage einer biologischen Metapher konstruiert ist, dann wird die Natur in der Biosozialität auf der Grundlage von Kultur modelliert werden, wobei ich Kultur als Praxis verstehe.« (Rabinow 2004: 139)

Ausgehend von Rabinows Hinweis auf Prozesse der gesellschaftlichen Autopoiesis wird klar, dass biomedizinische Innovationen nicht durch simple Technikkritik, die einen linearen Zusammenhang zwischen neuen Artefakten (hier: einem Gentest) und sozialen Wirkungen (hier: Stigmatisierung und Diskriminierung) annimmt, ausreichend zu analysieren sind. Vielmehr ist mit ambivalenten und widersprüchlichen Effekten $\mathrm{zu}$ rechnen: Es ist »Kultur als Praxis«, also komplexe Prozesse der Aneignung, Umformung, Re-Definition und Anwendung, in denen biomedizinische Artefakte in konkreten Situationen realisiert werden als Teil gesellschaftlicher Selbsteinwirkung.

\section{UND ICH? \\ ZUR Dialektik individualisierter Medizin}

Mit der Einführung der Begriffe »Autopoiesis«, »Kultur« und »Praxis« ist auf die Komplexität der Effekte verwiesen, wenn eine neue Technologie in einer Gesellschaft freigesetzt wird. Wenden moderne Gesellschaften etwa

\footnotetext{
5 Das Zitat geht weiter: »Natur wird mit Hilfe von Technik erkannt und neu hergestellt werden. Und sie wird schließlich artifiziell werden, genauso wie Kultur natürlich werden wird.«
} 
genetische Diagnoseverfahren auf ihre Bevölkerungen an, werden offensichtlich Wirkungen hervorgerufen, die sich nicht im Einfach-Vokabular einer deterministisch-mechanischen Folgen-Rhetorik beschreiben lassen (Soziale Konstruktion von Technologie). Auf diese Komplexitätseffekte versuchen die Begriffe Autopoiesis, Kultur oder Praxis zu reagieren: Sie verweisen auf rekursive Prozesse der Selbststeuerung gesellschaftlicher Systeme, auf Kreativität und soziale Innovativität ebenso wie auf die Eigen-Logik der Nutzung, die eigensinnige Aneignung durch Nutzer und ihre Selbstbeobachtung. Zugleich wird durch den Einsatz von Technologien in modernen Gesellschaften ein spezifischer Modus der Selbstreferentialität nahe gelegt: Denn solche sozialen Systeme müssen um Technologien entwickeln, implementieren und nutzen zu können - sich $»$ in ihren elementaren Operationen auf sich selbst beziehen [...] also auf die Elemente des Systems selbst, auf die Operationen des Systems selbst, auf die Einheit des Systems selbst.« (Luhmann 1987: 159) Dieses Konzept der Selbstreferenz - darauf hat die Darmstädter Philosophin Petra Gehring (2006) hingewiesen - überschreitet ein einfaches Verständnis von gesellschaftlicher Selbstorganisation in einem sehr entscheidenden Punkt: Selbstreferentialität beziehe sich eben nicht nur einfach auf Strukturen, sondern zusätzlich stets auch auf symbolische und kommunikative Operationen. (Gehring 2006: 344) Obwohl Luhmann selbst (1995: 398) den Begriff der Kultur als einen der schlimmsten, je gebildeten Begriffe charakterisierte, weil er ein adäquates Verständnis gesellschaftlicher Selbstbeschreibung und -referenz verhindere, erscheint aus sozialanthropologischer Perspektive die Charakterisierung solcher, durch technologische Entwicklungen vorangetriebener selbstreferentiellen Operationen als kulturelle Praxen mehr als nahe liegend. (Luhmann 2004; Beck 2009)

Hier ist momentan nur festzuhalten, dass die Begriffe Autopoiesis, Kultur und Praxis ent-simplifizieren, indem sie soziologische wie kulturanthropologische Gesellschaftstheorien anrufen und vor prognostischen wie deskriptiven Einfach- und Fehlverständnissen warnen. (Zimmerli 1990: 258) Entsprechend verweist das Wort Technologie nicht auf homogene »Dinge«, denen eine einfache Wirkung nachgesagt werden könnte (SOZIALE KONSTRUKTION VON TECHNOLOGIE), sondern es ist allenfalls ein Etikett, ${ }^{6}$ das in

6 Vgl. zu dieser Unterscheidung zwischen Etikett und Begriff Keating/Cambrosio 2003: »[a label] merely isolates a set of practices and objects without showing 
einer ersten provisorischen, noch vor-begrifflichen Annäherung auf heterogene, hochgradig zeitgebundene Arrangements von Dingen, Wissen, Praxen und Normen wie Normierungen geklebt werden kann (AKTEURNeTZWerk TheOrIE). Das wird gut deutlich, wenn man die Geschichte der isländischen Entdeckung weiter verfolgt: Was 2005 noch utopisch erschien, ist nach nur sechs Jahren "normalisiert» und kommerzialisiert. Mehrere Firmen - so etwa die vom Google-Gründer Sergey Brin finanziell unterstützte Firma »23andme« - bieten über das Internet Test-Kits an, mit denen Interessenten bequem zu Hause Speichelproben erfassen und zur genetischen Analyse per Post einschicken können. Angeboten wird, Dispositionen für Krankheiten wie Diabetes, potentielle Sucht-Abhängigkeiten, Besonderheiten des Stoffwechsels (wie etwa die Fähigkeit, Koffein schnell abzubauen) oder Nahrungsmittelunverträglichkeiten zu klären. Außerdem wird auf Grund des genetischen Profiles eine »ancestry-Map« erstellt, die individuelle Zugehörigkeit zu Populationen - Kunden können so erfahren, dass etwa einige ihrer Vorfahren südeuropäischer, afrikanischer oder nordeuropäischer Herkunft waren. ${ }^{7}$ (Pray 2008) Zum Geschäftsmodell gehört aber auch ein - tatsächlich revolutionärer - Ansatz: Die genetischen Daten der Kunden werden, wenn dazu ein Einverständnis vorliegt, zur weiteren Forschung verwandt. Das Versprechen ist hier, dass die Kunden doppelt profitieren können: einerseits, in dem sie altruistisch Forschung und Fortschritt fördern, andererseits, in dem sie von der Firma direkt benachrichtigt werden, wenn neue Erkenntnisse, die auf ihr persönliches genetisches Profil zutreffen, verfügbar sind. (Dolgin 2010)

Natürlich ist es eine triviale Feststellung, dass ein solches, simpel aussehendes Instrument wie das zum Preis von momentan 99 \$ verschickte »test-kit« nicht zureichend verstanden werden kann, wenn es als isoliertes technisches Artefakt wie etwa ein Fieberthermometer untersucht wird. Die Herausforderung besteht gerade darin, das im Artefakt Abwesende, nämlich die sehr komplexe Infrastruktur, die im Hintergrund in Gang gesetzt wird, und die moralischen Ökonomien - etwa die Mischung aus Eigennutz, dem

what holds them together and qulifies them as a set. As such, it cannot be used as an analytical resource.« (S. 326)

7 So wirbt etwa die Homepage von 23andme: »Gain insight into your traits, from baldness to muscle performance. Discover risk factors for 97 diseases. Know your predicted response to drugs, from blood thinners to coffee. And uncover your ancestral origins. « (www.23andme.com) 
Ziel, für die eigene und die Zukunft der Familie nützliche Prävention zu betreiben, und dem Gefühl, am Fortschritt teilhaben zu können - zu analysieren, deren in der Öffentlichkeit sichtbare Spitze das harmlos scheinende Kästchen ist. Es muss vermieden werden, Technik zu schnell auf das sichtbare und vergegenständlichte »Ding « einzugrenzen, mit dem sich ein überschaubarer, transparenter und in seinen Folgen absehbarer Umgang pflegen ließe.

Eine $\mathrm{zu}$ einfache, überwiegend materialistisch ausgerichtete Epistemologie verfehlt ihre analytischen Aufgaben gegenüber komplexen technologischen Artefakten ebenso wie symboltheoretische Ansätze zur »Dingbedeutsamkeit«, wie sie in Teilen der Ethnologie immer noch dominieren, weil sie die oben angesprochene Thematik der Selbstreferentialität vernachlässigen. Ebenso wenig hilfreich sind Ansätze, die davon ausgehen, dass Technik »Folgen« in einem einfachen Sinne hätte: So wäre es natürlich unsinnig, anzunehmen, dass die Veröffentlichung der Studie zum »Herzinfarkt-Gen« notwendig die Gründung von Patientengruppen zur Folge habe oder die Verfügbarkeit von »genetischen Dienstleistungen« wie der durch »23andme« offerierten zu einer durchgreifenden, massenhaften genetischen Transparenz führten. Ganz offensichtlich sind hier komplexere Prozesse der Koppelung und reflexiven Relationierung am Werk. Und auch die »Patienten « müssen - folgt man Rabinow in seinem Vorschlag - als Teil einer emergenten Kultur und als Träger gesellschaftlicher Praxisformen angesprochen werden und nicht als von Technik »Betroffene« und auf Innovationen »Reagierende«. Zudem - und dies ist der entscheidende Punkt - muss stets mit dialektischen Wirkungen technologischer Innovationen gerechnet werden: Angebote wie »23andme«, die auf den ersten Blick als Beispiel eines Trends hin zu einer »individualisierten Medizin« interpretiert werden können, haben durchaus das Potential, zu neuen Kollektivierungsformen beizutragen.

Ich gehe im Folgenden deshalb von drei sehr schlichten Prämissen aus: erstens, dass Technik kein »Gegenstand « oder »Ding« ist, sondern die Bedingungen für einen spezifischen Modus moderner Selbstreferentialität (i.S. der Selbsteinwirkung und der Selbstreflexivität) erzeugt; dass Technik zweitens keine »Folgen« in einem mechanistisch-deterministischen Sinne hat, sondern dass mit durchaus eigen-willigen und unvorhergesehenen Wirkungen gerechnet werden muss; und dass sich drittens über Technik nur sprechen lässt, wenn die Praxen der Akteure analysiert werden. Und ich 
gehe weiter davon aus, dass diese schlichten Prämissen recht komplexe theoretische Umstellungen und neue Perspektivierungen dessen nahe legen, was aus europäisch-ethnologischer Perspektive als »Technik« analysiert und als »Technikforschung« betrieben werden sollte. (Vgl. Beck 1997) Dazu möchte ich auf einige Begriffe zurückgreifen, die mir gut geeignet erscheinen, kultur- und sozialanthropologische bzw. europäisch-ethnologische Fragestellungen zu verfolgen.

\section{Dispositif/APPARATUS - PlattForm - INFRASTRUKTUR}

Ich möchte im Folgenden drei Begriffe knapp einführen, die den Debatten der internationalen Science and Technology Studies eine prominente Rolle spielen, und sie punktuell erweitern. Danach werde ich in einem weiteren Schritt - an einem Beispiel aus meiner eigenen Feldforschung - erläutern, warum ich sie als analytisch tauglich einschätze.

Der erste Begriff geht auf Michel Foucault zurück: er prägte das Konzept des dispositifs ab der Mitte der 70er Jahre, um damit soziale Arrangements der Kontrolle und Herrschaft zu bezeichnen. Dabei betonte er, dass ein dispositif eine große Zahl sehr unterschiedlicher, heterogener und oft widersprüchlicher Elemente in ein gemeinsames Netzwerk integriere. Ein solches Netzwerk, wie es etwa das um die öffentliche Gesundheitsvorsorge organisierte dispositif darstelle, umfasse demnach Diskurse um »Volksgesundheit«, Institutionen und architektonische Arrangements wie etwa Krankenanstalten und Asyle, politische Programme, Gesetze, Verwaltungsmaßnahmen, wissenschaftliche Aussagen etwa über das Kranke und Gesunde, philosophische, moralische und philanthrophische Grundannahmen über Rechte und wechselseitige Pflichten des Einzelnen gegenüber dem Kollektiv - kurz: es umfasse Gesagtes wie nicht-Gesagtes, das in einer spezifischen, zeittypischen Weise miteinander verknüpft werde (STS UND POLITIK).

Entscheidend ist, dass in diesem Konzept Materielles (Maschinen, Gebäude), Diskursives (Wahrheitsansprüche, wissenschaftliche Fakten, Moral) und Institutionelles (Politik und Verwaltung) nicht nur unter einem gemeinsamen Etikett versammelt werden, sondern dass Foucault einen systematischen Zusammenhang, ein Netzwerk dieser Elemente mit dem Begriff des 
dispositifs postuliert. Und dieses Netzwerk produziert Effekte, ähnlich wie eine komplexe Maschine, wie ein »apparatus«, um einen weiteren, oft synonym von Foucault verwendeten Begriff zu nennen. (Vgl. Rabinow/Rose 2003) So analysiert er die Gesundheitspolitik des 18. Jahrhunderts als eine Politik der sozialen Apparate, die ihre Objekte - etwa die Armen oder die gebärenden Frauen - strategisch auswählten, die Zielgruppen mit zunehmend wissenschaftlichen Methoden spezifizierten und sie dann der Kontrolle und ihrem »Schutz« unterwarfen. (Foucault 2001 (1994): 282-311.) Wichtig im hier diskutierten Zusammenhang ist vor allem, dass Foucault im Begriff des dispositifs konventionell voneinander getrennte Wirklichkeitsbereiche - also etwa Politik und Wissenschaft, Architektur und Moral -, die zudem üblicherweise von unterschiedlichen Wissenschaften behandelt werden, in einem analytischen Rahmen betrachtet (vgl. hierzu den Begriff der Heterogenität im Kapitel AKTEUR-NETZWERK THEORIE). Weiter ist entscheidend, dass der Begriff des dispositifs die Dynamik der jeweils geknüpften Netzwerke, ihre Zeitgebundenheit und ständige Transformation betont: Die von Foucault analysierten Kontroll-, Überwachungs- und Regulationsapparate sind hochgradig flexible, verteilte und flüchtige Gebilde, die sich weder räumlich noch zeitlich klar abgrenzen lassen.

Der zweite Begriff, den ich knapp skizzieren möchte, ist der der Plattform, der starke Parallelen mit Foucaults Konzept des dispositifs aufweist, sich aber auf den engeren Bereich von Wissenschaft und Technik konzentriert und im Gegensatz zu Foucaults Konzept des dispositif keine gesamte gesellschaftliche und kulturelle Formation zu erklären versucht. Ich entlehne ihn Überlegungen zur Entwicklung der Biomedizin, die die kanadischen Wissenschaftsanthropologen Peter Keating und Alberto Cambrosio, die beide an der McGill University in Montreal lehren, vor einigen Jahren vorlegten. $(2000 ; 2003)$ Sie analysieren die Entwicklung der Diagnostik für Blutkrebs seit Mitte des 20. Jahrhunderts, insbesondere die Veränderungen, die sich auf der Grundlage neuartiger molekularbiologischer Untersuchungsverfahren und durch die Verfügbarkeit immunologischer Analysetechniken in der Leukämie-Diagnostik ergeben haben.

Ähnlich wie Foucault behandeln Keating und Cambrosio die Bereiche von Wissenschafts- und Technikentwicklung in einem einheitlichen analytischen Rahmen und heben damit die nicht nur für weite Teile der sozialwissenschaftlichen Wissenschaftsforschung konventionelle Unterscheidung zwischen Wissenschaft, verstanden als Domäne der Wahrheitsproduktion, 
und Technik und Technologie, verstanden als Domäne der Anwendung von wissenschaftlichem Wissen, auf. Ihr Argument ist, dass ein Verständnis der modernen Biomedizin notwendig eine Betrachtungsweise erfordere, die Technik als einen konstitutiven Bestandteil epistemischer Praxen analysiere - und eben nicht nur als einfaches »Werkzeug« der Wissensproduktion. Ihre Perspektive wendet sich explizit sowohl gegen sozialdeterministische oder besser sozialkonstruktivistische Theorien der Wahrheitsproduktion als auch gegen technikdeterministische Sichtweisen bei der Analyse von Wissenschaft und Medizin. (Keating/Cambrosio 2003: 21)

Stattdessen prägen sie den Begriff der biomedizinischen Plattform, um damit die Kombination von heterogenen Elementen zu beschreiben, von denen jedes seine eigene Genealogie aufweist, die jedoch zu einer funktionierenden Form zusammengebracht werden. Es sind Techniken, Instrumente, Institutionen, chemische Reagentien, handwerkliche und kognitive Fertigkeiten, biologische Faktoren wie Zellproteine oder Gene, Repräsentationsweisen, diagnostische und prognostische Verfahren sowie ätiologische Verständnisse, die - einmal in Gang gesetzt - die Diagnose »Leukämie« produzieren können. Ähnlich wie bei Foucault erscheinen biomedizinische Plattformen bei Keating und Cambrosio als zugleich materielle wie diskursive Arrangements, als - in der Foucault'schen Diktion - Kombination von Gesagtem und Nicht-Sagbarem. Diese Plattformen koordinieren über einen Zeitraum hinweg medizinische und wissenschaftliche Praxen und fungieren als Werkbank, auf der schließlich auch neue Konzepte des Pathologischen erzeugt werden können. Keating und Cambrosio wollen mit ihrem Konzept insbesondere auch einige der Übertreibungen eines $\mathrm{zu}$ idealistisch geratenen Konstruktivismus korrigieren, der die Wirkungen von Technologien, die Materialität von Forschungsgegenständen oder die konstitutiven Effekte von Instrumenten vernachlässige. ${ }^{8}$ (Keating/Cambrosio 2000: 386)

Als einen dritten Begriff möchte ich kurz den der Infrastruktur einführen, der von der US-amerikanischen Wissensanthropologin Susan Leigh Star und ihrer Kollegin Karen Ruhleder für die Analyse eines computergestützten, virtuellen Laboratoriums für Wissenschaftler (eines »collaboratory« Systems) geprägt wurde. Diese Software sollte die Nutzer dabei unter-

8 Vgl. hierzu auch die Debatte zwischen den Richtungen Sociology of Scientific Knowledge und Akteur-Netzwerk Theorie (SociologY OF SCIENTIFIC KNOWLEDGE). 
stützen, ihre Forschungsergebnisse vorstellen und mit über 1400 Fachkollegen weltweit diskutieren zu können; sie stellte also die Infrastruktur für Kommunikation und Austausch zur Verfügung. (Star/Ruhleder 1996) Star und Ruhleder analysierten die Nutzungsmuster und -erwartungen der Benutzer dieses virtuellen Kommunikations- und Interaktionsraumes zwischen 1991 und 1994 auf der Grundlage teilnehmender Beobachtungen und Interviews. Es ist dabei durchaus bemerkenswert, dass die gefühlte historische Distanz zu dieser Epoche, in der Browser wie Mosaic oder Software wie Gopher gerade in ersten beta-Versionen verfügbar wurden, weit größer erscheint als die tatsächlich seitdem vergangenen Jahre dies erwarten liessen. Mag also der Gegenstand der Studie von Star und Ruhleder schon längst dem digitalen Vergessen anheim gefallen sein, das Konzept der Infrastruktur, das sie zur Analyse dieser Kommunikationsoberfläche entwickelten, und der Vorschlag, ein komplexeres Verständnis der Ökologien der Infrastruktur-Nutzung zu entwickeln, bleibt hoch aktuell.

Erhellend ist vor allem ihr Vorschlag, nicht zu fragen was eine Infrastruktur sei, sondern wann eine Infrastruktur sei. Dahinter steht eine praxistheoretische und performanztheoretische Sicht auf individuelles und kollektives Handeln sowie auf Wahrnehmung: Infrastruktur ist demnach etwas "that emerges for people in practice, connected to activities and structures." (Star/Ruhleder 1996: 112) Zentral ist, dass damit ein strikt relationales Konzept der Infrastruktur eingeführt wird: Infrastruktur ist Infrastruktur nur in Bezug auf konkrete, organisierte Praxisformen; sie ist eingebettet in soziale Arrangements und Routinen; sie ist für die Handelnden »transparent«, indem sie verborgene Hilfestellung bei Handlungen leistet; ihre Selbstverständlichkeit wird in Rahmen von Mitgliedschaften in "communities of practice« (Lave/Wenger 1992) erlernt; und Infrastrukturen besitzen in der Regel eine Reichweite, die die lokalen Kontexte der Praxisformen weit überschreitet. Diese relationale, praxistheoretische Perspektive unterscheidet den Infrastruktur-Begriff deutlich von Foucaults Konzept des dispositifs und ebenso von Keating und Cambrosios Plattform-Konzept.

Wichtig ist zudem, dass Star und Ruhleder hier einen etwas eigenwilligen Begriff der Transparenz einführen - er verweist bei ihnen darauf, dass die Infrastruktur im Handlungsprozess quasi unsichtbar (transparent) sei, dass sie sich nicht in den Aufmerksamkeitsfokus der Akteure schiebe. Star und Ruhleder gehen weiter davon aus, dass sich eine Infrastruktur dann »ereigne«, wenn die Spannungen zwischen dem Lokalen und dem Globalen 
aufgelöst würden: »That is, an infrastructure occurs when local practices are afforded by larger-scale technology, which can be used in a natural, ready-to-hand fashion ${ }^{9}$. It becomes transparent as local variations are folded into organizational changes, and becomes an unambiguous home - for somebody. This is not a physical location or a permanent one, but a working relation - since no home is universal.« (Star/Ruhleder 1996: 114)

Die Begriffe Dispositif, Plattform und Infrastruktur können ein analytisches Instrumentarium bereit stellen, mit dem Technik stets kontextualisiert, das heißt im Zusammenhang einer historisch spezifischen Situation, und eingebunden in konkrete Praktiken konkreter, sozial verorteter Menschen zum Thema gemacht wird. Gemeinsam ist diesen Begriffen ${ }^{10}$ weiter, dass sie materielle, diskursive, institutionelle und epistemische Dimensionen zugleich thematisieren, und damit vorschnelle Vergegenständlichungen oder materialistische Fehlschlüsse vermeiden helfen. Und die Begriffe verbindet schließlich, dass sie lokale Praktiken mit Prozessen höherer sozialer Ordnung verbinden - seien es nationalstaatliche Gesundheitsprogramme, internationaler Austausch in Wissenschafts-communities oder weltweit entwickelte und verfügbar gemachte biomedizinische Plattformen. Die aus der Perspektive der Europäischen Ethnologie interessante - wenn auch grammatikalisch etwas unbeholfen wirkende - Frage lautet dann: Wann ist für wen die Biomedizin? Und konkreter: Welche Prozesse der Infrastrukturierung - verstanden als eine Normalisierung von Biomedizin i.S. ihres Transparent-Werdens für die Handelnden - können beobachtet werden? Wie werden diese Prozesse mit den vorherrschenden, möglicherweise widersprüchlichen Dispositiven verknüpft? Wie verändern sich im Gebrauch dieser Infrastruktur, indem sich Nutzer in ein neues Verhältnis zu den technischen Optionen bringen, ihre Einstellungen, Normativitäten, Selbstverständnisse und Selbstverhältnisse? Wie lernen etwa Nutzer des

9 Dies wird im Sinne der Heidegger'schen Zuhandenheit (Heidegger 1993) konzipiert; entsprechend gehen Star und Ruhleder davon aus, dass Infrastrukturen in ihren tatsächlichen Dimensionen und in ihrer ganzen Komplexität erst wieder im Moment ihres Zusammenbruches sichtbar werden: sie büßen dann ihre Transparenz ein, werden opak, weil sie den Handelnden Widerstand entgegensetzen.

10 Die Begriffe taugen damit durchaus als Elemente einer »middle-range theory« i.S.v. Robert K. Merton's Diktum (1968: 39), dass zwischen Arbeitshypothesen und umfassend konzipierten Großtheorien Theorien mittlerer Reichweite entwickelt werden müssten, die den Austausch mit anderen Disziplinen ermöglichten. 
von 23 andme verschickten test-kits ihren Körper und dessen Zukunft anders zu sehen als sie dies bislang taten?

Es ist offensichtlich, dass diese Perspektive über eine Analyse von »Technikfolgen« nicht nur hinaus geht, sondern Untersuchungen konkreter Techniknutzungen erst auf praxistheoretische Füße zu stellen vermag. Um an einem Beispiel diese Perspektive zu verdeutlichen, möchte ich abschließend einige Aspekte dieser Normalisierung der Biomedizin an eigenem Feldforschungsmaterial skizzieren, das auf ein seit Frühjahr 2005 von mir in Zypern durchgeführtes Projekt zurückgeht. Aus Platzgründen muss die Darstellung des Materials ebenso wie die Interpretation sehr skizzenhaft erfolgen. Zypern führe ich aber auch deshalb als Beispiel an, weil sich an diesem Beispiel sehr gut jene Prozesse der Biosozialität analysieren lassen, die Rabinow als typisch für die »neue Gestalt« gesellschaftlicher Autopoiesis ansieht.

\section{KAPAIIKAKEIO IDPYMA - INFRASTRUKTUR EINES GLOBALISIERTEN ALtRUISMUS}

Die zypriotische Knochenmark-Spender-Datenbank ist als eine Realisation und Konkretisierung der gleichen biomedizinischen Plattform anzusehen, die Keating und Cambrosio beschreiben: Tiefgefrorene Gewebeproben von freiwilligen Spendern, Computer, Datenbanken, Leitzordner, Laborreagentien und -apparate, gut qualifizierte Labortechniker und Genetiker, die in den USA oder Europa ausgebildet wurden, ebenso wie internationale Forschungskooperationen, in die das Labor eingebunden ist, werden hier mobilisiert, um Leukämiekranke korrekt zu diagnostizieren und ihnen durch Vergleich mit den Datenbanken der internationalen KnochenmarksspenderDatenbanken eine lebensrettende Transplantation von Blut bildendem Knochenmark zu vermitteln.

Träger dieser Einrichtung ist die Karaiskakio Stiftung (K $\alpha \rho \alpha \_\kappa \alpha \kappa \varepsilon ı$ I $\rho v \mu \alpha)$, die von einem wohlhabenden Zyprioten namens Andreas Karaiskakios gegründet wurde, nachdem sein Sohn an Leukämie gestorben war, weil für ihn nicht rechtzeitig ein geeigneter Knochenmarksspender gefunden werden konnte. Als Direktor der Stiftung konnte ein junger zypriotischer Humangenetiker gewonnen werden, der in den USA an einem der renommiertesten Universitätskliniken als Spezialist für Blutkrebs-Diagnostik 
und Immunogenetik ausgebildet worden war. Die Labors ebenso wie die Administration der Karaiskakio-Stiftung, die inzwischen neun Angestellte in Administration und Labor hat, sind in einem kleinen ehemaligen Wohnhaus in der Hauptstadt der Insel untergebracht, das selbst einer vierköpfigen Familie große Kompromissfähigkeiten bezüglich ihrer Raumansprüche abverlangen würde, müsste sie dort wohnen. Jeder verfügbare Winkel des Hauses ist mit Aktenschränken, Computern, Laborbänken, Schreibtischen und Kühlschränken voll gestopft, in denen die Gewebeproben der Stiftung aufbewahrt werden.

In einem kleinen Hinterzimmer ist ein Labor um eine Analysemaschine herum gebaut, mit der bis zu 1.000 Gewebeproben pro Tag immunogenetisch typisiert werden können. Die Stiftung ist sehr stolz, dass in diesem kleinen Raum eines der modernsten und leistungsstärksten immunogenetischen Labore weltweit betrieben wird. Diese enorme Kapazität scheint auf der kleinen Insel im östlichen Mittelmeer völlig deplaziert, sie zu installieren war jedoch notwendig geworden, nachdem sich im März 2000, in der kurzen Zeitspanne von nur 25 Tagen, mehr als 57.000 Freiwillige als Knochenmarkspender registrieren ließen - immerhin fast 10\% der Gesamtbevölkerung. Diese überwältigende Welle der Hilfsbereitschaft war Ergebnis einer Pressemeldung in der griechisch-sprachigen Presse und folgenden Meldungen auch im türkischen Teil der Insel, die darauf hingewiesen hatten, dass die Stiftung Spender für zwei Leukämie-kranke Jungen suche, den griechischen Zyprioten Andreas Vassiliou und den türkischen Zyprioten Kemal Saraçoğlu. Über diese Suche wurde wegen der außergewöhnlichen Symbolik auch von der internationalen Presse berichtet. Tatsächlich gelang es schließlich unter maßgeblicher Beteiligung von Friedensaktivisten und gegen viele politische Widerstände der Regierungen beider Landesteile, die Spendenaktion zu einem überwältigendem Erfolg zu machen: Über die damals noch hermetische, streng überwachte und militärisch gesicherte Grenze hinweg, mit der der Kontakt zwischen der griechischsprachigen und der türkischsprachigen Bevölkerungsgruppe auf der Insel nahezu völlig unterbunden wurde, konnte ein Zeichen gegen die ethnische Spaltung beider Volksgruppen auf Zypern gesetzt werden.

Die Kampagne für die beiden Kinder sollte es - nach der Idee der Organisatoren - griechischen wie türkischen Zyprioten erlauben, in einer kritischen politischen Phase mit der Spende ihres Blutes ein Zeichen der Menschlichkeit zu setzen. Denn die Bemühungen der internationalen Ver- 
handlungsgruppe um UNO und EU, eine einvernehmliche Verhandlungslösung zwischen beiden Bevölkerungsgruppen noch vor dem EU-Beitritt des griechischen Teils der Insel zu erzielen, waren in den Wochen vor der Spendenkampagne einmal mehr in eine aussichtslos erscheinende Krise geraten. Während es für die Organisatoren und Aktivisten der griechisch- und türkisch-zypriotischen NGOs nicht zuletzt darum ging, mit der Spendenaktion ein an Symbolik kaum zu übertreffendes Signal für die Versöhnung zwischen den verfeindeten Gruppen auf der Insel zu setzen, betrachteten viele der Spender ihre Beteiligung als rein humanitären Akt, der sich gegen jede politische Indienstnahme verwahrte. Ein 38-jähriger Sport-Trainer, der lange in den USA gelebt hatte, erklärte so etwa auf meine Frage, ob es sich bei der Spenden-Aktion um einen außergewöhnlichen und gar symbolischen Akt gehandelt habe:

»Politiker mögen das als >besondere〈 Situation ansehen - ich denke aber nicht so. Politiker machen ihre Tricks, durch indirekte Handlungen. Symbolisch - vielleicht, weil die Insel hier getrennt ist, ja. Aber wenn du von einer humanitären Grundlage her denkst, dann denke ich nicht, dass es etwas Besonderes ist. Wenn jemand Hilfe braucht - warum würdest du die Hilfe verweigern, wenn du helfen kannst? Deshalb sind die Menschen auf beiden Seiten der Grenze zum Spenden gekommen. Arme Leute denken nicht an Politik. Warum sind sie gekommen? Hat sie jemand getrieben? Nein! « ${ }^{11}$

Alle Spender, mit denen ich Gespräche führen konnte, hatten sich sehr für die Herkunft »ihrer« strikt anonym bleibenden Empfänger interessiert und bei vielen war die anfängliche Überraschung, dass der Empfänger ihres Knochenmarks kein Zypriote, sondern ein Europäer, ein US-Amerikaner oder Australier war, einem deutlichen Stolz gewichen, Teil eines internationalen Netzwerkes der Humanität zu sein. Eine 30-jährige Verwaltungsangestellte etwa streicht die humanitäre Motivation heraus, die alle nationalen oder ethnischen Differenzen überwinde:

11 Interview L.K., 26.2.2005, Nikosia; ich danke Dr. Pavlos Costeas, Karaiskakeio Stiftung, und seinen Mitarbeitern für die Hilfe bei der Anbahnung der Interviews, die Zeit, die sie für Gespräche fanden, und die Geduld, den neugierigen Sozialwissenschaftler an ihren beengten Arbeitsplätzen zu dulden. 
»Anfangs war ich überrascht [dass der Empfänger Italiener war] aber es macht ja keinen Unterschied [...] Nationalität ist kein Thema. [...] ich kenne die Person nicht, und er kennt mich nicht - aber wir haben das gleiche Ziel.« ${ }^{12}$

Für die Stiftung gestaltete sich die überwältigende Beteiligung sowohl der griechisch- als auch der türkisch-sprachigen Zyprioten aus mehreren Gründen problematisch: Einerseits waren die Laborkapazitäten nicht darauf ausgerichtet, eine solche große Zahl von Gewebeproben in so kurzer Zeit daraufhin $\mathrm{zu}$ untersuchen, ob sich unter den Tausenden Freiwilligen ein immuno-kompatibler Spender befand; doch natürlich erwarteten Organisatoren wie die Öffentlichkeit schnelle Resultate. Zudem schien die Autonomie der Stiftung auch dadurch gefährdet, dass schon allein zur Konservierung der Gewebeproben auf Kühleinrichtungen staatlicher Einrichtungen zurück gegriffen werden musste. Staatliche Institutionen waren nur zu schnell bereit, »in die Bresche zu springen«. Und auch die Politisierung der Spenden durch Aktivisten und die nationale wie internationale Presse gefährdete schließlich die strikt neutrale Position, die die gemeinnützige Stiftung als notwendig erachtete, um unbehelligt über alle politischen Grenzen hinweg ihren humanitären Zielen nachgehen zu können. Hierzu ist sie auf die Kooperation oder zumindest die Duldung der politisch Herrschenden auf beiden Seiten der Grenze dringend angewiesen - eine zu große symbolische Aufladung schien dies zu gefährden.

Ich kann hier diesen Konflikten und Ambivalenzen nicht weiter nachgehen - wichtig ist im hier diskutierten Zusammenhang aber vor allem die Tatsache, dass Plattformen wie die gerade beschriebene offen sind für ganz unterschiedliche, symbolische, politische, rein humanitäre oder eigenwillige »In-Dienst-Nahmen«. Und entscheidend ist weiter, dass biomedizinische Plattformen von denjenigen, die sie betreiben, in ihren Wirkungen nicht kontrolliert werden können. So ist der eigentliche Zweck der biomedizinischen Plattform, wie er von Wissenschaftlern und Technikern entwickelt und als internationaler Standard in Kliniken durchgesetzt wurde, die Diagnose unterschiedlicher Leukämien, vor allem aber der Feststellung der immunologischen Kompatibilität potentieller Spender. Entsprechend setzt die Stiftung das Labor als eine Maschine zur Herstellung immunologischer Differenz und Ähnlichkeit ein: sie produziert Singularitäten - immunotypi-

12 Interview E.T., 24.2.2005, Nikosia. 
sierte Kranke und immunkompatible Spender. Von den politisch motivierten Organisatoren der Spendenkampagne hingegen wurde die Plattform zur Demonstration politischer Gleichheit und einer verbindenden Humanität genutzt - zur Produktion von Kollektivitäten.

\section{INFRASTRUKTURIERUNG - INTRASTRUKTURIERUNG}

Diese Unterschiede sind keine Frage von »richtigem Gebrauch» oder »Missbrauch«. Aus der Perspektive der Europäischen Ethnologie geht es darum, kulturelle Muster und Kosmologien (Sahlins 1996) zu rekonstruieren, die unterschiedliche, sozial akzeptable Nutzungsweisen orientieren. Bei beiden Nutzungsweisen - der symbolisch-politischen ebenso wie bei der rein humanitären, medizinischen - geht es um gute und ehrenhafte Zwecke, doch sind diese Zwecke eingebunden in unterschiedliche Dispositive, in soziale Apparate, die zwar letztlich beide Leben retten oder Lebensbedingungen verbessern wollen, die in diesem Fall jedoch um Ressourcen und gesellschaftliche Aufmerksamkeit konkurrieren. Das eine Dispositiv ist organisiert um die Frage der Gesundheit und Krankenfürsorge, das andere Dispositiv um die Frage der politischen Freiheit, der Gleichheit und der Menschenrechte. Um die Metapher der Plattform aufzunehmen: $\mathrm{Zu}$ ihr führen viele Wege und viele Wege führen von ihr weg; ebenso, wie sie heterogene Fakten und Artefakte mit ganz unterschiedlichen Genealogien verbindet, verknüpft eine biomedizinische Plattform unterschiedliche Zwecke mit verschiedenen Mitteln innerhalb eines soziotechnischen Rahmens. Das heißt, sie ermöglicht sehr unterschiedliche und teils gegenläufige oder widersprüchliche, jedoch gleich legitime Praxisformen.

Auch aus Sicht der Spender und Empfänger stellt sich die Situation jeweils radikal anders dar. Die Frage, wann für sie Infrastruktur ist, und welche Infrastruktur dies jeweils ist, ist eng an ihre konkreten Positionen und sozialen Situationen gebunden. So haben die an Leukämie Erkrankten keinen direkten Kontakt mit den Laborwissenschaftlern, die die Art ihres Blutkrebses diagnostizieren oder die ihre immunologischen Werte feststellen; ihr Berührungspunkt mit dem biomedizinischen System ist der behandelnde Klinikarzt. Und von diesem Arzt erfahren sie - wenn ihnen das Glück günstig ist -, dass irgendwo auf der Welt ein passender Spender für 
sie gefunden wurde. Im Falle einer erfolgreichen Transplantation folgen Tests, medikamentöse Einstellungen und weitere Behandlungen - denn die Kranken müssen stets damit rechnen, dass das Transplantat abgestoßen werden kann. (Vgl. Quenzler 2005) Für sie ist die immunologische Plattform vollkommen transparent - sie verschwindet im Hintergrund klinischer Praxen und ihres andauernden, chronischen Leidens. Ähnlich transparent stellt sich die Plattform auch für die Spender dar, wenn sie sich erstmals registrieren und danach zur Spende aufgefordert werden: Sie werden von einer Spenderorganisation kontaktiert und nach weiteren immunologischen Tests wird ihnen in einem kurzen, schnell verheilenden Eingriff blutbildendes Gewebe entnommen; schon am nächsten Tag können sie in der Regel ihr normales Leben wieder aufnehmen.

Ganz anders ist jedoch die Situation zwischen Spendern und Empfängern: Hier herrscht - entsprechend internationaler Bioethik-Richtlinien strikte Anonymität: So wissen die Spender in der Regel nicht, ob der Empfänger ihrer Spende überlebt hat oder nicht. Wie in meinen Gesprächen mit Knochenmarksspendern immer wieder deutlich wurde, empfinden diese die Anonymität und das erzwungene Nicht-Wissen als ungerechte psychische Belastung und Zumutung. Denn für sie stellt der Akt der Spende vor allem ein spirituelles Verhältnis zum Empfänger her: fast alle Spender berichten mir von der Existenz eines geistigen Bandes zum Empfänger ihrer Zellen, sei es, dass sie Vorahnungen hatten, die die Gesundheit des jeweiligen Empfängers betrafen und die sich später als zutreffend herausstellten. Oder das Verhältnis zwischen Spendern und Empfängern wird von ihnen als eines der Kreation verstanden - wie mir ein Spender sagte: »Sein Vater gab ihm sein erstes Leben, ich gab ihm sein zweites. ${ }^{13}$ Was die von mir befragten Spender mit großer Intensität spüren, ist eine - in beiderlei Wortsinne zu verstehende - Verbindlichkeit zwischen Spender und Empfänger, eine spirituelle Nähe, die Raum, Zeit, Geschlecht und fremde ethnische Zugehörigkeit überbrückt. (Vgl. Beck 2011) Bemerkenswert ist, dass die hochtechnologische Basis dieser Verbindung, die immunologisch-biotechnologische Plattform, die diese Nähe erst ermöglicht hat, für sie völlig verschwindet. Gegenüber dieser positiv erlebten Transparenz erfahren sie die bioethischen Regulationsmechanismen, die Teil des »apparatus« sind, als völlig destruktiv: Der Zwang zur Anonymität verhindere, dass sie ihren »natürlichen«

13 Interview A.A., 25.2.2005, Limassol. 
Impulsen nachgeben und dem »natürlichen« Verhalten nachgehen zu können - nämlich ihr neues Geschwister, den Menschen, der in ihrer Überzeugung »Fleisch von ihrem Fleische ist«, auch körperlich in den Arm nehmen und spüren zu können. Hier wird die Infrastruktur plötzlich »sichtbar«, sie verliert ihre Transparenz und wird Gegenstand von Beschwerden und Protest, Versuchen, die Anonymitätsregeln zu umgehen, oder schließlich Anlass für Enttäuschung und Resignation.

Der Infrastrukturierung, also dem Verschwinden der biotechnologischen Plattform im Moment ihrer Nutzung durch Spender und Empfänger, entspricht eine komplexe Intrastrukturierung: mit der neuen Technologie werden Bedingungen und Regeln für neue Verbindlichkeiten geschaffen, die oft als »natürlich« erscheinen, und es entsteht die kulturelle Herausforderung, zivilisatorisch neue Mischungsverhältnisse von Hochtechnologie und Emotion zu erproben. Dies ist eine der Dimensionen der Biosozialität, wie sie mit biomedizinischen Plattformen in der Gesellschaft frei gesetzt wird - eine neue Form der Autopoiese. Für die Nutzer erfordert dies neue Arten der (Selbst-)Reflexion; und für die Sozialwissenschaft, die diese Wirkungen analysiert, erfordert dies ein komplexes Verständnis des Zusammenhanges zwischen Technik und Technologie - verstanden als Infrastruktur - einerseits und andererseits Nutzungsmustern und Handlungsorientierungen. Analog zu dem in einem Teil der Science and Technology Studies modisch gewordenen Begriff der Ko-Produktion schlage ich vor, diesen Zusammenhang symmetrisch - aber auch nicht-deterministisch - als Ko-Konstitutionsverhältnis zu untersuchen (vgl. Giddens 1988). Zu analysieren wären mithin die entstehenden transnationalen, teilweise marktförmig organisierten Zonen des humanitären und altruistischen Tausches, die widerstreitenden Dispositive sowie die sich dort bildenden moralischen Praxen. 


\section{LITERATUR}

Beck, Stefan (1997): Umgang mit Technik. Kulturelle Praxen und kulturwissenschaftliche Forschungskonzepte, Berlin: Akademie Verlag.

Beck, Stefan (2004): »Fryst altruism, varm solidaritet och kall etik. Om en biobank på Cypern«. In: Susanne Lundin (Hg.), En ny kropp. Essäer om medicinska visioner och personliga val, Lund: Nordic Academic Press, S. 43-76.

Beck, Stefan (2009): »Vergesst Kultur - wenigstens für einen Augenblick! Oder: Zur Vermeidbarkeit der kulturtheoretischen Engführung ethnologischen Forschens«. In: Sonja Windmüller/Beate Binder/Thomas Hengartner (Hg.), Kultur - Forschung. Zum Profil einer volkskundlichen Kulturwissenschaft (Studien zur Alltagskulturforschung, Bd. 6), Berlin: Lit Verlag, S. 48-68.

Beck, Stefan (2011): »Staging bone marrow donation as a ballot. Reconfiguring the Social and the Political using Biomedicine in Cyprus«. Body \& Society 17(2/3), Special Issue: Medical Migrations, S. 93-119.

Cornelis, Marilyn C./El-Sohemy, Ahmed/Kabagambe, Edmond K./Campos, Hannia (2006): »Coffee, CYP1A2 Genotype, and Risk of Myocardial Infarction«. Journal of the American Medical Association 295, S. 1135-1141.

Dolgin, Elie (2010): »Personalized investigation«. Nature Medicine 16(9), S. 953-955.

Foucault, Michel (1994): »The Birth of Social Medicine«. In: Paul Rabinow/ Nikolas Rose (Hg.), The Essential Foucault. Selections from Essential Works of Foucault 1954-1984, New York: Pantheon Books, S. 319-337.

Foucault, Michel (2001): In Verteidigung der Gesellschaft. Vorlesungen am Collège de France (1975-76), Frankfurt/M.: Suhrkamp.

Gehring, Petra (2006): „Selbstorganisation und Selbstreferenz. Vom wissenschaftlichen Selbstorganisationsparadigma zu Luhmanns Theorie sozialer Systeme«. In: Vec, Miloš/Marc-Thorsten Hütt/Alexandra M. Freund (Hg.), Selbstorganisation. Ein Denksystem für Natur und Gesellschaft, Köln: Böhlau, S. 341-354.

Giddens, Anthony (1988): Die Konstitution der Gesellschaft. Grundzüge einer Theorie der Strukturierung. Mit einer Einführung von Hans Joas, Frankfurt/M.: Suhrkamp. 
Hall, Edward (2004): »Spaces and Networks of Genetic Knowledge Making: the >Genetisation« of Heart Disease«. Health \& Place 10, S. 311318.

Hall, Edward (2005): »The `Genetisation` of Heart Disease: a Network Analysis of the Production of New Genetic Knowledge«. Social Science and Medicine 60, S. 2673-2683.

Heidegger, Martin (1993): Sein und Zeit, Tübingen: Max Niemeyer Verlag. Helgadottir, Anna et al. (2005): »A Variant of the Gene Encoding Leukotriene A4 Hydrolase Confers Ethnicity-specific Risk of Myocardial Infarction«. Nature Genetics 10. 11., advance online publication: doi:10.1038/ng1692.

Helgason, Agnar et al. (2000): »mtDNA and the Origin of the Icelanders: Deciphering Signals of Recent Population History«. American Journal of Human Genetics 66(3), S. 999-1016.

Keating, Peter/Cambrosio, Alberto (2000): »Biomedical Platforms «. Configurations 8, S. 337-387.

Keating, Peter/Cambrosio, Alberto (2003): Biomedical Platforms: Realigning the Normal and the Pathological in Late-Twentieth-Century Medicine, Cambridge/MA: MIT Press.

Lave, Jean/Wenger, Etienne (1992): Situated Learning: Legitimate Peripheral Participation, Cambridge: University of Cambridge Press.

Luhmann, Niklas (1987): »Archimedes und wir. Interviews, hg. von Dirk Baecker/Georg Stanizek, Berlin: Merve.

Luhmann, Niklas (1995): Die Kunst der Gesellschaft. Frankfurt/M.: Suhrkamp.

Luhmann, Niklas (2004): „Sinn, Selbstreferenz und soziokulturelle Evolution«. In: Günter Burkart/Gunter Runkel (Hg.), Luhmann und die Kulturtheorie, Frankfurt/M.: Suhrkamp, S. 241-289.

Merton, Robert K. (1968): »On Sociological Theories of the Middle Range«. In: Robert Merton (Hg.), Social Theory and Social Structures, New York, S. 39-73.

Pálsson, Gísli/Harđardóttir, Kristín (2002): »For Whom the Cell Tolls: Debates about Biomedicine«. Current Anthropology 43(2), S. 271-301.

Pálsson, Gísli/Rabinow, Paul (1999): »Iceland. The Case of a National Human Genome Project«. Anthropology Today 15(5), S. 14-18.

Pálsson, Gísli/Rabinow, Paul (2001): »The Icelandic Genome Debate«. Trends in Biotechnology 19(5), S. 166-171. 
Pray, Leslie (2008): »Direct-to-Consumer Genetic testing: 23andme, DNA Direct and Genelex«. Nature Education 1(1), http://www.nature.com/ scitable/topicpage/dtc-genetic-testing-23andme-dna-direct-and-674, abgerufen am 24. August 2011.

Quenzler, Miriam (2005): »Langzeitpatienten und ihre Ärzte: eine partnerschaftliche Beziehung. Das Beispiel Knochenmarkstransplantation«. In: Gisela Welz/Gesa Heinbach/Nadja Losse/Annina Lottermann/Sabrina Mutz (Hg.), Gesunde Ansichten. Wissensaneignung medizinischer Laien, Frankfurt/M.: Kulturanthropologie Notizen, Bd. 74, S. 115-132.

Rabinow, Paul (2004): Anthropologie der Vernunft. Studien zu Wissenschaft und Lebensführung, Frankfurt/M.: Suhrkamp.

Rabinow, Paul/Rose, Nikolas (2003): »Introduction - Foucault Today«. In: Paul Rabinow/Nikolas Rose (Hg.), The Essential Foucault: Selections from the Essential Works of Foucault, 1954-1984, New York: New Press, S. vii-Xxxv.

Rose, Hilary (2001): The Commodification of Bioinformation: The Icelandic Health Sector Database. With a foreword by Marilyn Strathern, London: The Wellcome Trust.

Sahlins, Marshall (1996): »The Sadness of Sweetness. The Native Anthropology of Western Cosmology«. Current Anthropology 37, S. 395-428.

Sigurdsson, Skuli (2001): »Yin-yang genetics, or the HSD deCode controversy«. New Genetics and Society 20, S. 103-117.

Star, Susan Leigh/Ruhleder, Karen (1996): »Steps toward an Ecology of Infrastructure: Design and Access for large Information Spaces«. Information Systems Research 7(1), S. 111-134.

Vince, Gaia (2005): »Heart risk gene hits African Americans hardest $«$. newScientist,11.11., online publication: http://www.newscientist.com/ article/dn8300-heart-risk-gene-hits-african-americans-hardest.html, abgerufen am 24. August 2011.

Zimmerli, Walther Ch. (1990): „Wie viel Akzeptanz erträgt der Mensch? Bemerkungen zu den Hintergründen der Technikfolgenabschätzung«. In: Ernst Kistler/Dieter Jaufmann (Hg.), Mensch - Technik - Gesellschaft, Opladen, S. 247-260. 
